

\title{
Virtual series-system models of imperfect repair
}

\author{
Ibrahim Nafisah $^{\mathrm{a}}$, Mansour Shrahili ${ }^{\mathrm{a}}$, Naif Alotaibi ${ }^{\mathrm{b}}$, Phil Scarf ${ }^{\mathrm{c} *}$ \\ ${ }^{a}$ Department of Statistics and Operations Research, King Saud University, Riyadh, Kingdom of Saudi Arabia \\ ${ }^{b}$ Department of Mathematics and Statistics, Imam Mohammad Ibn Saud Islamic University, Kingdom of \\ Saudi Arabia
}

${ }^{c}$ Salford Business School, University of Salford, Manchester, UK

*corresponding author: email: p.a.scarf@salford.ac.uk

\begin{abstract}
Novel models of imperfect repair are fitted to classic reliability datasets. The models suppose that a virtual system comprises a component and a remainder in series. On failure of the component, the component is renewed, and on failure of the remainder, the component is renewed and the remainder is minimally repaired. It follows that the repair process is a counting process that is the superposition of a renewal process and a Poisson process. The repair effect, that is, the extent to the system is repaired by renewal of the component, depends on the relative intensities of the superposed processes. The repair effect may be negative, when the intensity of the part that is a renewal process is a decreasing function. Other special cases of the model exist (renewal process, Poisson process, superposed renewal process and homogeneous Poisson process). Model fit is important because the nature of the model and corresponding parameter values determine the effectiveness of maintenance, which we also consider. A cost-minimizing repair policy may be determined provided the cost of preventive-repair is less than the cost of corrective-repair and the repairable part is ageing. If the remainder is ageing, then policy needs to be adapted as it ages.
\end{abstract}

Keywords: renewal process; counting process; reliability; maintenance; multi-component system

\section{Introduction}

Much research has sought to model imperfect repair of a multi-component system in a way that captures a repair effect that lies somewhere between "as good as new" (perfect repair) and "as bad as old" (minimal repair). At the ends of this spectrum lie the renewal process (Ross, 1996) and the non-homogeneous Poisson process (Cox and Lewis, 1966). In between, and thus capturing imperfect repair, lie mixed processes (Brown and Proschan, 1983; Kallen, 2011; Doyen, 2014) virtual age-reduction models (Kijima and Sumita, 1986; Kijima, 1989; Jack, 1998; Tanwar et al., 2014), geometric processes (Lam, 1988; Wang and Pham, 1996; Wu and Clements-Croome, 2006; Wu and Scarf, 2015; Zhang et al., 2015; Wu and Wang, 2018), hazard-reduction models (Doyen and Gaudoin, 2004; Percy and Alkali, 2006; Percy et al., 2010), virtual component models (Wu and Scarf, 2017); and generalizations of these (Doyen et al., 2017). All these attempt to model multicomponent systems parsimoniously.

Models with few parameters are desirable because failure data are scarce (Scarf, 1997) and often non-existent (e.g. Desa and Christer, 2001). As a consequence, maintenance policy is imprecisely specified (Baker and Scarf, 1995). While a component-wise model of imperfect repair of multi-component systems, in which repair corresponds to renewal of a particular component, is conceptually simple, such a model requires knowledge of individual component reliabilities. 
Component dependencies (e.g. Do et al., 2018) add further difficulties. Many components have never failed and most rarely fail (e.g. Akbarov et al., 2008). Thus, research that seeks to model the repair-effect, either directly or indirectly, with as few parameters as possible is important. This paper continues this tradition.

A focus of these parsimonious models is estimation of the repair effect. This is directly parameterized within the age- and hazard-reduction models, and indirectly parameterized in the models described in this paper. Estimation of the repair effect is challenging because data are typically scarce and subjective elicitation from maintenance engineers who carry out repairs is compromising (Zhang and Wang, 2014). It may be better therefore for repair models to indirectly specify the repair effect, so that it is a property of the model (a function of parameters) rather than a parameter itself. Then it can be indirectly estimated. Thus, one of the objectives of this paper is the indirect estimation of the repair effect. We also allow that this repair effect may be negative, whereby, for example, a poor-quality spare-part is used or a fault is induced (Scarf and Cavalcante, 2012) at preventive maintenance.

The repair effect is estimated using a model that we outline here. Conceptionally, the model posits a series system with an unknown number $m$ of components. By unknown here, we mean that $m$ is a parameter about which we have no information. Each component generates events that occur in time according to a counting process (Cox and Lewis, 1966). These events are typically failures but need not be so in general. This is convenient because failures are often ambiguously defined and what is observed are the times of maintenance interventions, which we term repairs. At each event, the system is repaired, so that the events are triggers for repair. On repair of the system, we suppose that a component is replaced with a new (age-zero) identical component, but the component replaced does not necessarily correspond to the component that fails, or more generally that generates the repair event. If these components are indeed different, then the component that generates the repair event is unaffected by the event. If they are one and the same, then it is replaced. In this way, we suppose the system is a virtual system consisting of a virtual component, which is the component that is always replaced on repair, and a virtual sub-system, which is the collection of the other virtual components in the system. The latter is the remainder of the system. We use this virtual-system terminology to distinguish the system from a 1-out-of- $m$ series systemmodels of repair of series systems (e.g. Smith and Dekker, 1997) usually assume that the component that fails is replaced. Further, we suppose that each virtual component corresponds to a counting process.

The fundamental ideas for this type of model are described in Wu and Scarf (2017). In our paper here we take a number of further steps. First, we show that the virtual series-system model can be formulated as a superposition of a renewal process and a nonhomogeneous Poisson process. Second, we fit the model (and special cases of it) to a number of classic datasets from the reliability literature. In so doing, we consider carefully the goodness-of-fit of the models, a critical activity given the scarcity of data in real situations. We then use the models to show how the repair effect can be specified and estimated. Finally, we show how the models can be used to inform maintenance policy decision-making and discuss their implications for practice.

The layout of the paper then corresponds to the order of these developments. However, first are some preliminaries about counting processes that will be needed for the model specification in Section 3. The subsections in Section 3 present the models and their properties. The final subsection of Section 3 describes parameter estimation for the model by the method of maximum 
likelihood. Section 4 describes the classic datasets we use, the parameterization of the renewal process and Poisson process elements of the model, and the results of the model fitting. Section 5 is concerned with maintenance policy and the repair effect. We finish with conclusions in the final section.

\section{Preliminaries}

First we state some preliminary definitions and results that are well-known (Cox and Lewis, 1966). The number of events that arise in an interval $[0, \tau]$ is in general a counting process $N(\tau)$ whose behaviour is determined by the intensity function

$$
\lambda\left(t \mid \mathcal{F}_{t-}\right)=\lim _{\delta t \rightarrow 0} \frac{\operatorname{Pr}\left(N(t+\delta t)-N(t) \geq 1 \mid \mathcal{F}_{t-}\right)}{\delta t},
$$

where $\mathcal{F}_{t-}$ is the history (filtration) of the process up to time $t . \lambda\left(t \mid \mathcal{F}_{t-}\right)$ is an intensity function of a counting process if and only if $\Lambda(t)=\int_{0}^{t} \lambda\left(u \mid \mathcal{F}_{u^{-}}\right) \mathrm{d} u$ exists for all $t \in[0, \tau]$, is positive and is an increasing function in $t$. Essentially this means $\lambda($.) must be positive and defined everywhere on the positive real line. $\Lambda(t)$ is called the cumulative intensity function (CIF).

If the intensity function does not depend on the history $\left(\lambda\left(t \mid \mathcal{F}_{t-}\right)=\lambda(t)\right)$ and at most one event can occur at any time $t$, then the counting process is a Poisson process. If the intensity function is a constant $(\lambda(t)=\lambda)$ then the counting process is a homogeneous Poisson process. If the intensity function depends only on the time since the last event $\left(\lambda\left(t \mid \mathcal{F}_{t-}\right)=h\left(t-T_{N(t-)}\right)\right)$ then the counting process is a renewal process. Consequently, the renewal process is characterized by the distribution $F_{X}$ of the time since the last event, $X=t-T_{N(t-)}$, and $h$ is the hazard function of this distribution $\left(h(x)=F_{X}^{\prime}(x) /\left\{1-F_{X}(x)\right\}\right)$.

The homogeneous Poisson process is a renewal process and $X$ is exponentially distributed with mean $1 / \lambda$, and $h_{X}(x)=F_{X}^{\prime}(x) /\left\{1-F_{X}(x)\right\}=\lambda \exp (-\lambda x) / \exp (-\lambda x)=\lambda$.

Our next preliminary point concerns the addition (superposition) of counting processes. Let $N_{1}(\tau)$ and $N_{2}(\tau)$ be counting processes. Then their superposition $N_{\mathrm{S}}(\tau)=N_{1}(\tau)+N_{2}(\tau)$ is a counting process, and if $\lambda_{1}\left(t \mid \mathcal{F}_{1, t-}\right)$ does not depend on $\mathcal{F}_{2, t-}$ and vice versa (independence), then $N_{\mathrm{S}}(\tau)$ has intensity function $\lambda_{1}\left(t \mid \mathcal{F}_{1, t-}\right)+\lambda_{2}\left(t \mid \mathcal{F}_{2, t-}\right)$.

\section{Virtual series-system models}

\subsection{Partially renewing process}

We describe two models. In the first, it is always the oldest component in the virtual system that is replaced on repair. In the second model, notionally, it is the youngest.

The virtual system (VS) is 1-out-of- $m$ system with $m$ identical and independent virtual components. Each virtual component corresponds to a counting process. We do not attempt to make a correspondence between these $m$ virtual components of the virtual system and $m$ components of a real, multi-component system. Thus, the virtual system idea is conceptual. We use the term virtual to qualify component and system deliberately, in a way that is analogous to using the term virtual in the virtual age models of imperfect repair.

The 1-out-of- $m$ system structure implies that the virtual system is the superposition of these $m$ independent, identical counting processes. Suppose events (repairs) have occurred at times $t_{1}, t_{2}, \ldots, t_{i-1}, \ldots(i>m)$. Let $h_{\mathrm{C}}($.$) be a hazard function.$ 


$$
\lambda_{\mathrm{S}}(t)=\left\{\begin{array}{lr}
m h_{\mathrm{C}}(t), & t<t_{1}, \\
\sum_{j=1}^{i-1} h_{\mathrm{C}}\left(t-t_{j}\right)+(m-i+1) h_{\mathrm{C}}(t), & t_{i-1} \leq t<t_{i}, 1<i \leq m, \\
\sum_{j=i-m}^{i-1} h_{\mathrm{C}}\left(t-t_{j}\right), & t_{i-1} \leq t<t_{i}, \quad m<i,
\end{array}\right.
$$

is the intensity function of a counting process.

Proof. For all $t>0, \Lambda_{\mathrm{S}}(t)=\int_{0}^{t} \lambda_{\mathrm{S}}(u) \mathrm{d} u$ is defined, positive and increasing in $t$ because $h_{\mathrm{C}}(t)$ is defined and positive because it is a hazard function. End of proof.

The intensity function $\lambda_{\mathrm{S}}(t)$ characterises the virtual system, but the proof is not instructive, so we make some remarks. The construction of $\lambda_{\mathrm{S}}(t)$ is such that before the first repair (event) $\left(t<t_{1}\right)$ the $m$ virtual components are each aged $t$ with identical hazards $h_{\mathrm{C}}(t)$. After the first repair, and at a time when the number of virtual components is at least as big as the number of repairs (second line on right hand side), then some virtual components have been replaced (renewed) and some have not. The latter collectively contribute $(m-i+1) h_{\mathrm{C}}(t)$ to the system intensity, the former $h_{\mathrm{C}}\left(t-t_{1}\right)+h_{\mathrm{C}}\left(t-t_{2}\right)+\ldots$ When the number of repairs exceeds the number of virtual components $(m<i)$, the ages of the $m$ virtual components (arguments of the hazards) correspond to the times since the $m$ most recent repairs. In this way, we interpret the model as one in which the oldest component in the virtual system is replaced at a repair.

At steady-state $(t \rightarrow \infty)$, if $h_{\mathrm{C}}(t)$ is increasing with $t$ (IFR) (Barlow et al., 1996), then $\lambda_{\mathrm{S}}(t)<m h_{\mathrm{C}}\left(x_{m}\right)$, where $x_{m}$ is the time since the $m$ th most recent repair, and so the counting process is stationary (in the sense that $\lambda_{\mathrm{S}}(t)$ never increases without limit). We might call this a partially renewing process. The size of $m$ determines the extent to which the system is partially renewed at each repair, and the system is always somewhat better than old on repair. If $h_{\mathrm{C}}(t)$ is decreasing with $t$ (DFR), then again the counting process is stationary at steady-state, but here on repair the system will tend to be worse than old.

\subsection{Superposition of a renewal process and a Poisson process}

Consider now the notion that the youngest component is replaced on repair. Then the same component is always replaced on repair. The next proposition describes the system intensity function.

Proposition 2. Let $t=t_{0}=0$. Then the function

$$
\lambda_{\mathrm{S}}(t)=h_{\mathrm{C}}\left(t-t_{i-1}\right)+(m-1) h_{\mathrm{C}}(t), \quad t_{i-1} \leq t<t_{i}, \quad i=1,2, \ldots,
$$

is the intensity function of a counting process.

Proof. For all $t>0, \Lambda_{\mathrm{S}}(t)=\int_{0}^{t} \lambda_{\mathrm{S}}(u) \mathrm{d} u$ is defined, positive and increasing in $t$ because $h_{\mathrm{C}}(t)$ is defined and positive because it is a hazard function. End of proof.

Here the same virtual component is replaced at every repair; this is the renewing hazard $h_{\mathrm{C}}\left(t-t_{i-1}\right)$. The other $m-1$ virtual components have ages that are unchanged on repair; this is the non-renewing hazard $(m-1) h_{\mathrm{C}}(t)$.

If $h_{\mathrm{C}}($.$) is a decreasing function (DFR), so that a repair returns the system to a state that is$ "worse than old", then $\lambda_{\mathrm{S}}(t) \rightarrow h_{\mathrm{C}}(x)+\lambda$ as $\left.t \rightarrow \infty\left(\lim _{t \rightarrow \infty} h_{\mathrm{C}}(t)=\lambda \geq 0\right)\right)$, where $x$ is the time 
since last repair, and so the counting process is a renewal process in the limit (at steady-state). If $\lambda>0$, then the limiting process with intensity (2) is the superposition of a renewal process (RP) and a homogeneous Poisson process (HPP) with intensity $\lambda$. When $h_{\mathrm{C}}($.$) is an increasing function$ (IFR), if it increases without limit $\left(h_{\mathrm{C}}(t) \rightarrow \infty\right.$ as $\left.t \rightarrow \infty\right)$, then $\lambda_{\mathrm{S}}(t)$ increases without limit and the process is non-stationary. If $h_{\mathrm{C}}(t) \rightarrow \lambda$ as $t \rightarrow \infty$, then $\lambda_{\mathrm{S}}(t) \rightarrow h_{\mathrm{C}}(x)+\lambda$, as $t \rightarrow \infty$, and again at steady-state the virtual system process is the superposition of a renewal process and a homogeneous Poisson process. This then suggests a modification to the model in which we set

$$
\lambda_{\mathrm{S}, 2}(t)=h_{\mathrm{C}}\left(t-t_{i-1}\right)+\lambda_{\mathrm{R}}(t), \quad t_{i-1} \leq t<t_{i}, \quad i=1,2, \ldots,
$$

corresponding to a virtual system comprising a virtual, renewing component with hazard $h_{\mathrm{C}}($.$) and$ the remainder of the system with intensity function $\lambda_{\mathrm{R}}(t)$. This model is introduced as a failure model in $\mathrm{Wu}$ and Scarf (2017), wherein when the component fails it is replaced, and when the remainder fails, the component is replaced and the remainder is minimally repaired. The virtual, renewing component is called the virtual component (VC). The remainder, which is minimally repaired, is called the virtual sub-system (VSS). The VC and the VSS form the virtual system (VS). We shall use these terms in this paper. The model (3) is a superposition of a renewal process (RP) and a nonhomogeneous Poisson process (NHPP).

In the special case of (3) with

$$
\lambda_{\mathrm{S}, 1}(t)=h_{\mathrm{C}}\left(t-t_{i-1}\right)+\lambda, \quad t_{i-1} \leq t<t_{i}, \quad i=1,2, \ldots,
$$

the VC is a renewal process (RP) and the VSS is a homogeneous Poisson process (HPP), and so the VS is an RP - the superposition of an RP and an HPP is another RP. The partially renewing process (1) is a simplification of a second model introduced in Wu and Scarf (2017).

For the model $\lambda_{\mathrm{S}, 2}(t)$, equation (3), if $h_{\mathrm{C}}(t)$ is DFR, then repairs return the system to a state that is worse than just before repair (worse than old). If $h_{\mathrm{C}}(t)$ is IFR, then repairs return the system to a state that is better than just before repair (better than old but not as good as new). The repair effect - the extent to which repair improves (or worsens) the system-depends on $h_{\mathrm{C}}(t)$. If $\lambda_{\mathrm{R}}(t)$ is large, repairs are frequent but the reduction (if $h_{\mathrm{C}}(t)$ is IFR) in intensity at each repair will be small. When $\lambda_{\mathrm{R}}(t)$ is small, repairs are infrequent but the reduction (if $h_{\mathrm{C}}(t)$ is IFR) in intensity at each repair will be large.

\subsection{Further remarks}

The partially renewing process (1) might be generalized so that $r$ from $m$ virtual components are renewed on failure, although data are unlikely to distinguish a "1-from-3" model from a " 2 -from-6" model. Nonetheless the hazard function can be formulated in this case.

Replacement of the youngest virtual component always on repair when $h_{\mathrm{C}}($.$) is DFR may be$ an interesting model. Software reliability might follow such a process. On release (repair), a new version has new code that is the least reliable part of the software and the remainder of the code has been minimally repaired (debugged).

When $h_{\mathrm{C}}()=$.$h (a constant, non-time-dependent), then \lambda_{\mathrm{S}}(t)$ in $(1)$ is a constant intensity and the repair process is a homogeneous Poisson process (HPP). When $h_{\mathrm{C}}()=h,. \lambda_{\mathrm{S}, 2}(t)$ in (3) is the intensity of a superposition of an HPP and an NHPP, which is an NHPP. This suggests that $\lambda_{\mathrm{S}, 2}(t)$ provides a more flexible model than $\lambda_{\mathrm{S}}(t)$ in (1). Also, the latter partially renewing process is likely to be difficult to distinguish from an HPP (at estimation) because of its stationarity. Further, the 
non-stationarity of (2) - its ageing behavior - is linked to the hazard of the VC. This essentially makes the model considerably less flexible than (3).

So, in the remainder of the paper, we focus the model $\lambda_{\mathrm{S}, 2}(t)$, equation (3), and its special case $\lambda_{\mathrm{S}, 1}(t)$, equation (4).

\subsection{Parameterization}

When $h_{\mathrm{C}}($.$) is specified with two parameters (e.g. Weibull hazard h(t)=\left(\beta_{1} / \alpha_{1}\right)\left(t / \alpha_{1}\right)^{\beta_{1}-1}$ (Lawless, 1983), $\lambda_{\mathrm{S}, 1}(t)$ (equation 4 ) has three parameters.

A natural parameterization for remainder of the system (the non-renewing virtual sub-system, VSS $)$ is $\lambda_{\mathrm{R}}(t)=\left(\beta_{2} / \alpha_{2}\right)\left(t / \alpha_{2}\right)^{\beta_{2}-1}$, which corresponds to the power-law non-homogeneous Poisson process (e.g. Rigdon and Asit, 1989), and can model degradation of e.g. mechanical systems $\left(\beta_{2}>1\right)$ and improvement of e.g. software systems $\left(\beta_{2}<1\right)$. Then $\lambda_{\mathrm{S}, 2}(t)$ (equation 3) has four parameters. When $\lambda_{\mathrm{R}}(t)=0$ for all $t$, then the model reduces to a renewal process (RP) (2 parameters)

Notice that intensity (2) can model an ageing VS with three parameters ( $m$ plus two for the RP).

\subsection{Mean time to next repair and mean time between repairs}

In this sub-section we derive the mean time to next repair of the virtual system, and, when it exists, the mean time between repairs.

First consider the model with intensity function $\lambda_{\mathrm{S}, 2}(t)$, equation (3). Given a repair at time $t *$ , the time to next repair $Z=\min (X, Y)$, where $X$ is the random variable with hazard function $h_{\mathrm{C}}(z)$ and $Y$ is the random variable with hazard function $\lambda_{\mathrm{R}}\left(t^{*}+z\right)$. As an aside, we can see that the model is related to a competing risks model (e.g. Crowder, 2012).

Then, the mean time to the next repair, $E\{\min (X, Y)\}$ can be obtained as follows. Denote the probability density and reliability (survival) functions of $X$ by $f_{\mathrm{C}}(x)$ and $S_{\mathrm{C}}(x)$ and the probability density and reliability functions of $Y$ by $f_{\mathrm{R}, t^{*}}(y)$ and $S_{\mathrm{R}, t^{*}}(y)$. Then the probability density function of $Z=\min (X, Y)$ is given by

$$
f_{Z}(z)=f_{C}(z) S_{\mathrm{R}, t^{*}}(z)+f_{\mathrm{R}, t^{*}}(z) S_{\mathrm{C}}(z) .
$$

This is because $\operatorname{Pr}\{\min (X, Y) \leq z\}=1-\operatorname{Pr}(X>z) \operatorname{Pr}(Y>z)$ and differentiating both sides with respect to $z$ gives (5). Then, given a repair at $t *$, the mean time to the next repair is

$$
E\{\min (X, Y)\}=\int_{0}^{\infty} z\left\{f_{C}(z) S_{\mathrm{R}, t^{*}}(z)+f_{\mathrm{R}, t^{*}}(z) S_{\mathrm{C}}(z)\right\} \mathrm{d} z .
$$

When the VSS is HPP, $Y \sim \operatorname{Ex}(\lambda)$ (exponentially distributed) and $\lambda$ does not depend on $t^{*}$, and so the mean time between repairs (MTBR) exists and is given by is a constant:

$$
\operatorname{MTBR}=E\left\{\min \left(X, Y_{\exp }\right)\right\}=\int_{0}^{\infty} z\left\{f_{C}(z)+\lambda S_{\mathrm{C}}(z)\right\} \exp (-\lambda z) \mathrm{d} z
$$

Finally, if $X=T$ is a constant and $\lambda_{\mathrm{R}}(t)=\lambda$, then $Z=\min \left(T, Y_{\exp }\right)$ and the repair process looks like that arising from an age based replacement policy (Barlow and Hunter, 1960) that is applied to a non-ageing system. 


\subsection{Parameter estimation}

Proposition 4. Suppose that the repair times are $t_{1}, \ldots, t_{n}$, the virtual system is new (all virtual components are new) at $t=t_{0}=0$, the virtual system is observed over $\left[0, t_{n+1}\right]$, and the intensity function $\lambda_{\mathrm{S}, 2}(t ; \theta)$ is parameterized by $\theta$. Then the log-likelihood function for $\theta$ is given by

$$
\begin{aligned}
l\left(\theta, t_{1}, \ldots, t_{n}\right)=\sum_{k=1}^{n} \log \left\{h_{\mathrm{C}}\left(t_{k}-t_{k-1}\right)+\lambda_{\mathrm{R}}\left(t_{k}\right)\right\} \\
\quad-\sum_{k=1}^{n+1} \int_{0}^{t_{k}-t_{k-1}} h_{\mathrm{C}}(t) \mathrm{d} t-\int_{0}^{t_{n+1}} \lambda_{\mathrm{R}}(t) \mathrm{d} t .
\end{aligned}
$$

Proof. The likelihood function for a counting process with intensity $\gamma(t)$, and failure times as defined, is given by $\prod_{i=1}^{n} \gamma\left(t_{i}\right) \exp \left\{-\int_{0}^{t_{n+1}} \gamma(t) \mathrm{d} t\right\}$ in general (Andersen et al., 1993). Hence (7) follows on substitution of $\lambda_{\mathrm{S}, 2}(t)$. End of proof.

Notice that when $\lambda_{\mathrm{R}}(t)=\lambda\left(\mathrm{RP}+\mathrm{HPP}\right.$ model with intensity $\left.\lambda_{\mathrm{S}, 1}(t)\right)$, the log-likelihood in (7) simplifies to

$$
l\left(\theta, t_{1}, \ldots, t_{n}\right)=\sum_{k=1}^{n} \log \left\{h_{\mathrm{C}}\left(t_{k}-t_{k-1}\right)\right\}+n \lambda-\sum_{k=1}^{n+1} \int_{0}^{t_{k}-t_{k-1}} h_{\mathrm{C}}(t) \mathrm{d} t-\lambda t_{n+1} .
$$

Setting $\lambda=0$ gives the likelihood for the RP. When $h_{\mathrm{C}}()=$.0 , so the model is the HPP, expression (8) reduces to $l=n \log \lambda-\lambda t_{n}$. Equivalently, $h_{\mathrm{C}}()=$.$h (constant) and \lambda=0$ gives the same model (HPP).

Competing models can be compared using AIC (Akaike, 2011). Model fit can be checked by plotting the cumulative number of repairs and the fitted cumulative intensity function, and by comparing the MTBR with that implied under the fitted model, provided it exists.

\section{Model fitting}

\subsection{Classic datasets}

Now we fit models with intensities $\lambda_{\mathrm{S}, 1}(t)$ and $\lambda_{\mathrm{S}, 2}(t)$ to well-known datasets and compare with HPP and RP in each case, on basis of AIC. We cite the originator of the dataset in each case. The datasets are given in Table 1, in order of analysis: photocopier (Baker, 1996), Halfbeak (Ascher and Feingold, 1984), Grampus (Lee, 1980), 180t dump-truck (Coetzee, 1996), air-conditioners 7909, 7912, 7913, 7914 (Proschan, 1963). These datasets have been analyzed by many (e.g. Akman and Raftery, 1986; Baker, 1991; Vaurio, 1999; Phillips, 2000, 2001; Pulcini, 2001; Hong and Pai, 2006; Caroni, 2010; Fuqing and Kumar U, 2012; Gámiz and Lindqvist, 2016; Doyen et al., 2017; Syamsundar and Kumar, 2017), and feature in classic analyses in text books (e.g. Crowder et al., 1991; Meeker and Escobar, 1998; Blischke and Murthy, 2000).

\subsection{Specification of the hazard functions}

We now specify the hazard functions of the VC and the VSS. We use a Weibull hazard for both the $\mathrm{VC}$, so that $h_{\mathrm{C}}(t)=\left(\beta_{1} / \alpha_{1}\right)\left(t / \alpha_{1}\right)^{\beta_{1}-1}$, and the VSS, so that $\lambda_{\mathrm{R}}(t)=\left(\beta_{2} / \alpha_{2}\right)\left(t / \alpha_{2}\right)^{\beta_{2}-1}$. In this way the lifetime of the $\mathrm{VC}$ (which is always renewed on repair) is Weibull distributed, and failures of the VSS, which are minimally repaired, arise according to the power-law non-homogeneous Poisson process. The full model, which we denote "RP+NHPP", has 4 parameters.

The reduced model with $\beta_{2}=1$, so that $\lambda_{\mathrm{R}}(t)=\lambda=1 / \alpha_{2}$ (constant), which we denote "RP+HPP", has three parameters and its mean time between repairs (MTBR) is 


$$
\operatorname{MTBR}=\int_{0}^{\infty} z\left\{\frac{\beta_{1}}{\alpha_{1}}\left(\frac{z}{\alpha_{1}}\right)^{\beta_{1}-1}+\frac{1}{\alpha_{2}}\right\} \exp \left\{-\left(\frac{z}{\alpha_{1}}\right)^{\beta_{1}}-\frac{z}{\alpha_{2}}\right\} \mathrm{d} z .
$$

When the virtual system is just the VC $\left(\lambda=1 / \alpha_{2}=0\right)$, the model is a renewal process, has 2 parameters, and the MTBR is the mean of a Weibull distribution: $\alpha_{1} \Gamma\left(1+1 / \beta_{1}\right)$.

When the virtual system is just the VSS (no renewing component) and the VSS is HPP, the model has one parameter $\left(\alpha_{2}\right)$ and the MTBR is $\alpha_{2}$.

Suppose the VS is subject to the maintenance policy and the VSS is HPP: on failure of the VSS, minimally repair the VSS and replace the VC; and when the VC reaches age $\alpha_{1}$, then replace the VC. Then the MBTR is $E\left\{\min \left(\alpha_{1}, Y\right)\right\}$, where $Y \sim E x(\lambda)$. Thus

$$
\operatorname{MTBR}=\int_{0}^{\alpha_{1}} \exp \left\{-z / \alpha_{2}\right\} \mathrm{d} z
$$

\begin{tabular}{|c|c|c|c|c|c|c|c|c|c|c|c|c|c|c|}
\hline \multicolumn{3}{|c|}{ photocopier } & \multicolumn{2}{|c|}{ Halfbeak } & \multicolumn{2}{|c|}{ Grampus } & \multicolumn{4}{|c|}{ 180t dump-truck } & \multirow{2}{*}{$\begin{array}{r}7909 \\
90\end{array}$} & \multirow{2}{*}{$\begin{array}{r}7912 \\
23\end{array}$} & \multirow{2}{*}{$\begin{array}{r}7913 \\
97\end{array}$} & \multirow{2}{*}{$\begin{array}{r}7914 \\
50\end{array}$} \\
\hline 7 & 411 & 768 & 1382 & 21603 & 860 & 11511 & 78 & 13265 & 17968 & 20432 & & & & \\
\hline 8 & 419 & 831 & 2990 & 21658 & 1258 & 11575 & 158 & 13508 & 17984 & 20433 & 100 & 284 & 148 & 94 \\
\hline 9 & 461 & 868 & 4124 & 21688 & 1317 & 12100 & 331 & 13673 & 18175 & 20434 & 160 & 371 & 159 & 196 \\
\hline 58 & 470 & 875 & 6827 & 21750 & 1442 & 12126 & 381 & 13780 & 18443 & 20698 & 346 & 378 & 163 & 268 \\
\hline 84 & 475 & 925 & 7472 & 21815 & 1897 & 12368 & 523 & 14443 & 18458 & 21460 & 407 & 498 & 304 & 290 \\
\hline 86 & 482 & 937 & 7567 & 21820 & 2011 & 12681 & 620 & 14501 & 18667 & 21543 & 456 & 512 & 322 & 329 \\
\hline 98 & 505 & 940 & 8845 & 21822 & 2122 & 12795 & 664 & 14656 & 18669 & 21584 & 470 & 574 & 464 & 332 \\
\hline 104 & 509 & 943 & 9450 & 21888 & 2439 & 13399 & 1805 & 14906 & 18701 & 21602 & 494 & 621 & 532 & 347 \\
\hline 104 & 527 & 946 & 9794 & 21930 & 3203 & 13668 & 1817 & 14983 & 18723 & 21645 & 550 & 846 & 609 & 544 \\
\hline 112 & 533 & 946 & 10848 & 21943 & 3298 & 13780 & 2068 & 15004 & 18822 & 21706 & 570 & 917 & 689 & 732 \\
\hline 113 & 552 & 952 & 11993 & 21946 & 3902 & 13877 & 3253 & 15062 & 18860 & 21762 & 649 & 1163 & 690 & 811 \\
\hline 119 & 555 & 954 & 12300 & 22181 & 3910 & 14007 & 4489 & 15072 & 18922 & 21867 & 733 & 1184 & 706 & 899 \\
\hline 121 & 561 & 957 & 15413 & 22311 & 4000 & 14028 & 4725 & 15136 & 18935 & 21912 & 777 & 1226 & 812 & 945 \\
\hline 127 & 561 & 993 & 16497 & 22634 & 4247 & 14035 & 4961 & 15206 & 18945 & 21914 & 836 & 1246 & 1018 & 950 \\
\hline 127 & 575 & 1013 & 17352 & 22635 & 4411 & 14173 & 5138 & 15247 & 18960 & 21937 & 865 & 1251 & 1100 & 955 \\
\hline 194 & 587 & 1077 & 17632 & 22669 & 4456 & 14173 & 5200 & 15700 & 18961 & 21938 & 983 & 1263 & 1154 & 991 \\
\hline 195 & 603 & 1099 & 18122 & 22691 & 4517 & 14449 & 5278 & 15714 & 18979 & 21939 & 1008 & 1383 & 1185 & 1013 \\
\hline 212 & 622 & 1108 & 19067 & 22846 & 4899 & 14587 & 5711 & 15972 & 19013 & 21951 & 1164 & 1394 & 1401 & 1152 \\
\hline 216 & 630 & 1125 & 19172 & 22947 & 4910 & 14610 & 6400 & 16186 & 19032 & 21954 & 1474 & 1397 & 1447 & 1362 \\
\hline 229 & 635 & 1135 & 19299 & 23149 & 5676 & 15070 & 6444 & 16284 & 19034 & 21982 & 1550 & 1411 & 1558 & 1459 \\
\hline 229 & 639 & $\bullet \bullet \bullet$ & 19360 & 23305 & 5755 & $\bullet \bullet \bullet \bullet$ & 6677 & 16329 & 19169 & $\bullet \bullet \bullet \bullet \bullet$ & 1576 & 1482 & 1597 & 1489 \\
\hline 230 & 646 & & 19686 & 23491 & 6137 & & 7999 & 16425 & 19184 & & 1620 & 1493 & 1660 & 1512 \\
\hline 266 & 651 & & 19940 & 23526 & 6221 & & 8001 & 16605 & 19201 & & 1643 & 1507 & 1678 & 1525 \\
\hline 267 & 651 & & 19944 & 23774 & 6311 & & 8489 & 16723 & 19416 & & 1705 & 1518 & 1869 & 1539 \\
\hline 279 & 673 & & 20121 & 23791 & 6613 & & 9000 & 16731 & 19455 & & 1835 & 1534 & 1887 & $\bullet \bullet \bullet \bullet$ \\
\hline 292 & 684 & & 20132 & 23822 & 6975 & & 9086 & 16797 & 19525 & & 2043 & 1624 & 2050 & \\
\hline 300 & 692 & & 20431 & 24006 & 7335 & & 10262 & 16859 & 19595 & & 2113 & 1625 & 2074 & \\
\hline 301 & 693 & & 20525 & 24286 & 8158 & & 10817 & 17090 & 19601 & & 2214 & 1641 & $\bullet \bullet \bullet$ & \\
\hline 308 & 695 & & 21057 & 25000 & 8498 & & 11062 & 17305 & 19613 & & 2422 & 1693 & & \\
\hline 317 & 698 & & 21061 & 25010 & 8690 & & 11082 & 17484 & 19643 & & $\bullet \bullet \bullet$ & 1788 & & \\
\hline 324 & 709 & & 21309 & 25048 & 9042 & & 11086 & 17510 & 19671 & & & $\bullet \bullet \bullet$ & & \\
\hline 335 & 712 & & 21310 & 25268 & 9330 & & 11122 & 17511 & 19713 & & & & & \\
\hline 337 & 714 & & 21378 & 25400 & 9394 & & 11534 & 17536 & 19785 & & & & & \\
\hline 352 & 722 & & 21391 & 25500 & 9426 & & 12031 & 17621 & 19801 & & & & & \\
\hline 384 & 731 & & 21456 & 25518 & 9872 & & 12339 & 17703 & 19937 & & & & & \\
\hline 393 & 742 & & 21461 & $\bullet \bullet \bullet \bullet$ & 10191 & & 12733 & 17809 & 19990 & & & & & \\
\hline
\end{tabular}

Table 1. Times of repairs; photocopier in days; others in hours 


\subsection{Results}

Parameter estimates and AICs are shown in Table 2. Where the RP+NHPP is best model so far, we also fit the NHPP. The best models on the basis of minimum AIC are shaded. Observed cumulative number of failures and fitted cumulative intensity functions (CIF) are shown in Figures 1-5. Where there is some interesting behavior the CIF is shown "zoomed-in".

Table 2. Maximum likelihood estimates and standard errors, all datasets, and AIC and fitted (model) mean time between repairs (MTBR).

\begin{tabular}{|c|c|c|c|c|c|c|c|c|c|c|c|}
\hline & & $\alpha_{1}$ & s.e. & $\beta_{1}$ & s.e. & $\alpha_{2}$ & s.e. & $\beta_{2}$ & s.e. & AIC & $\begin{array}{l}\text { Fitted } \\
\text { MTBR }\end{array}$ \\
\hline photocopier & $\mathrm{RP}$ & 11.781 & 1.418 & 0.914 & 0.072 & - & - & - & - & 649.5 & 12.3 \\
\hline$n=92$ & HPP & - & - & - & - & 12.33 & 1.29 & - & - & 648.3 & 12.3 \\
\hline \multirow[t]{2}{*}{$M T B R=12.3$} & $\mathrm{RP}+\mathrm{HPP}$ & 66.19 & 1.23 & 46.96 & 29.15 & 12.62 & 1.34 & - & - & 647.4 & 12.3 \\
\hline & $\mathrm{RP}+\mathrm{NHPP}$ & 0.919 & 0.10 & 44.45 & 24.40 & 8.49 & 4.51 & 66.1 & $*$ & 648.8 & \\
\hline Halfbeak & $\mathrm{RP}$ & 251.1 & 49.9 & 0.631 & 0.06 & - & - & - & - & 949.3 & 355.3 \\
\hline$n=71$ & HPP & - & - & - & - & 359.4 & 42.68 & - & - & 979.6 & 359.4 \\
\hline \multirow[t]{3}{*}{$M T B R=359.4$} & $\mathrm{RP}+\mathrm{HPP}$ & 245.7 & 50.2 & 0.618 & 0.06 & $5.073 e 6$ & 8388 & - & - & 935.8 & 354.2 \\
\hline & $\mathrm{RP}+\mathrm{NHPP}$ & 1599 & 443 & 1.499 & 0.48 & $1.201 \mathrm{e} 4$ & 2264 & 5.384 & 1.25 & 918.7 & \\
\hline & NHPP & - & - & - & - & 5443.3 & 1010 & 2.759 & 0.32 & 927.9 & \\
\hline Grampus & $\mathrm{RP}$ & 265.8 & 38.43 & 0.971 & 0.103 & - & - & - & - & 742.6 & 269.3 \\
\hline$n=56$ & HPP & & & & & 269.1 & 36.0 & & & 740.7 & 269.1 \\
\hline \multirow[t]{2}{*}{$M T B R=269.1$} & $\mathrm{RP}+\mathrm{HPP}$ & 1320 & 19.5 & 69.00 & 73.9 & 273.7 & * & - & - & 741.2 & 271.4 \\
\hline & $\mathrm{RP}+\mathrm{NHPP}$ & 1100 & 1433 & 0.991 & 0.703 & 730.1 & 305.4 & 1.24 & $*$ & 744.8 & \\
\hline 180t dump-t & $\mathrm{RP}$ & 132.4 & 17.8 & 0.693 & 0.047 & - & - & - & - & 1541.6 & 169.1 \\
\hline$n=128$ & HPP & & & & & 171.7 & 15.2 & & & 1575.4 & 171.7 \\
\hline \multirow{3}{*}{$M T B R=171.7$} & $\mathrm{RP}+\mathrm{HPP}$ & 135.2 & 18.5 & 0.690 & 0.047 & 9783 & 4212 & - & - & 1543.7 & 168.7 \\
\hline & $\mathrm{RP}+\mathrm{NHPP}$ & 333.9 & 92.8 & 0.727 & 0.086 & 9232 & 1951 & 4.743 & 1.041 & 1515.1 & \\
\hline & NHPP & & & & & 1004 & 279 & 1.572 & 0.139 & 1554.7 & \\
\hline 7909 & RP & 90.90 & 13.83 & 1.293 & 0.18 & - & - & - & - & 315.7 & 84.0 \\
\hline$n=29$ & HPP & - & - & - & - & 83.52 & 15.51 & - & - & 316.7 & 83.5 \\
\hline \multirow[t]{2}{*}{$M T B R=83.5$} & $\mathrm{RP}+\mathrm{HPP}$ & 310.1 & 2.297 & 124.7 & 129.4 & 72 & $*$ & - & - & 316.5 & 87.3 \\
\hline & $\mathrm{RP}+\mathrm{NHPP}$ & 99.74 & 18.23 & 1.324 & 0.20 & 503 & $*$ & 0.718 & 0.55 & 319.6 & \\
\hline 7912 & RP & 54.62 & 12.37 & 0.853 & 0.12 & - & - & - & - & 307.8 & 59.3 \\
\hline$n=30$ & HPP & - & - & - & - & 59.60 & 10.88 & - & - & 307.3 & 59.6 \\
\hline \multirow[t]{3}{*}{$M T B R=59.6$} & $\mathrm{RP}+\mathrm{HPP}$ & 261.0 & 0.002 & $1.357 \mathrm{e} 05$ & $*$ & 57.45 & 10.0 & - & - & 292.7 & 56.8 \\
\hline & $\mathrm{RP}+\mathrm{NHPP}$ & 261 & 0.000 & $1.000 \mathrm{e} 06$ & $*$ & 266.6 & 99.3 & 1.745 & 0.32 & 285.3 & \\
\hline & NHPP & & & & & 186.7 & 80.3 & 1.505 & 0.28 & 304.9 & \\
\hline 7913 & $\mathrm{RP}$ & 79.91 & 14.37 & 1.123 & 0.17 & - & - & - & - & 291.9 & 76.6 \\
\hline$n=27$ & HPP & - & - & - & - & 76.83 & 14.79 & - & - & 290.4 & 76.8 \\
\hline \multirow[t]{2}{*}{$M T B R=76.8$} & $\mathrm{RP}+\mathrm{HPP}$ & 213.7 & 5.14 & 46.99 & 60.6 & 82.40 & 16.8 & - & - & 288.3 & 76.0 \\
\hline & $\mathrm{RP}+\mathrm{NHPP}$ & 213.6 & 42.5 & 45.10 & 0.2 & 70.00 & $*$ & 0.952 & 49.6 & 290.2 & \\
\hline 7914 & $\mathrm{RP}$ & 64.80 & 13.63 & 1.025 & 0.16 & - & - & - & - & 251.7 & 64.1 \\
\hline$n=24$ & HPP & - & - & - & - & 64.14 & 13.10 & - & - & 249.7 & 64.1 \\
\hline \multirow[t]{3}{*}{$M T B R=64.1$} & $\mathrm{RP}+\mathrm{HPP}$ & 210.0 & 0.002 & $1.229 \mathrm{e} 5$ & $1.68 \mathrm{e} 04$ & 69.70 & 15.2 & - & - & 234.7 & 66.3 \\
\hline & $\mathrm{RP}+\mathrm{NHPP}$ & 210.0 & 0.002 & $7.318 \mathrm{e} 6$ & $*$ & 143.0 & 50.4 & 1.200 & 0.22 & 225.9 & \\
\hline & NHPP & & & & & 82.91 & 51.82 & 1.088 & 0.22 & 251.6 & \\
\hline
\end{tabular}

* standard error not estimable

We now comment on the fitted models and parameter estimates, taking each dataset in turn.

For the photocopier data, we might select RP+HPP as the best model here, and Figure 1 shows the cumulative number of failures against time and the fitted cumulative intensity function for this model. However, for the RP+HPP, $\hat{\beta}_{1}$ is very large, and so $\hat{\alpha}_{1}$ is somewhat close to the boundary of the parameter space, $\max _{i=1, \ldots, n}\left(x_{i}\right)$, the largest inter-failure time. We can see this inter-failure time in Figure 1b, and the rapidly increasing hazard associated with it. This dataset is also complicated by frequent zero-valued inter-failure times, most likely due to imprecision in the timing of failure. We assumed the inter-failure times were 0.5 (half a day) in this case. So, some very short 
inter-failure times, which suggest hyper-exponentiality $\left(\hat{\beta}_{1}<1\right.$ in RP), and an absence of very long inter-failure times, which would account for the very large $\hat{\beta}_{1}$ in the RP+HPP, make drawing conclusions from these results difficult.

The results for the Halfbeak data are clearer cut. The AIC and the standard errors of the best fitting model support this claim. The RP+NHPP is the best model, suggesting that the system comprises a renewing part that is IFR $\left(\hat{\beta}_{1}=1.5\right)$ and remainder that is ageing $\left(\hat{\beta}_{2}=5.4\right)$ and rapidly so in later life (Figure 2a). The renewing IFR component is apparent in the plotted cumulative intensity (Figure 2b).

For the Grampus, failures appear completely random and the system is not ageing (HPP). This is confirmed to an extent by $\hat{\beta}_{1} \approx 1$ in the RP model. Here the MLE procedure for RP+HPP is encountering difficulty (very flat likelihood function) because this model is over-parameterized in these circumstances. Essentially, when the data arise from a Poisson process, parameters in a model that is a superposition of processes will be difficult to resolve.

a)

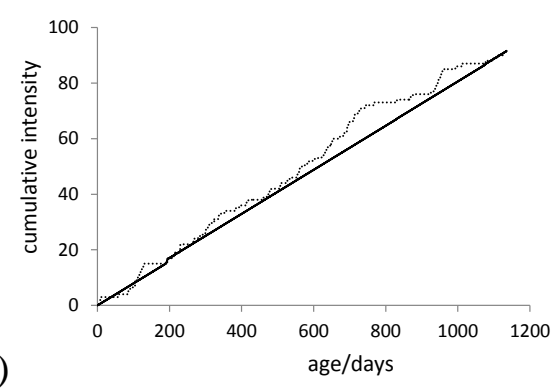

b)

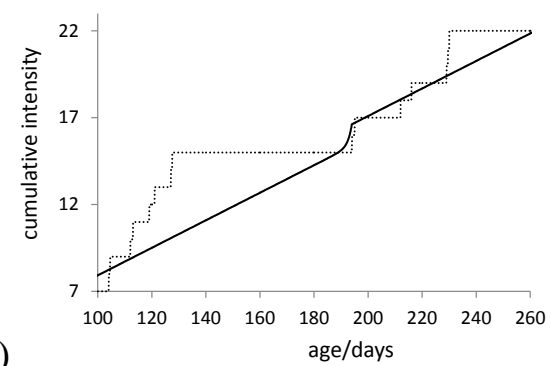

Figure 1. Photocopier repair data: cumulative number of repairs (dotted line) and fitted (RP+HPP) cumulative intensity function (solid line). a) all data; b) zoomed in.

a)

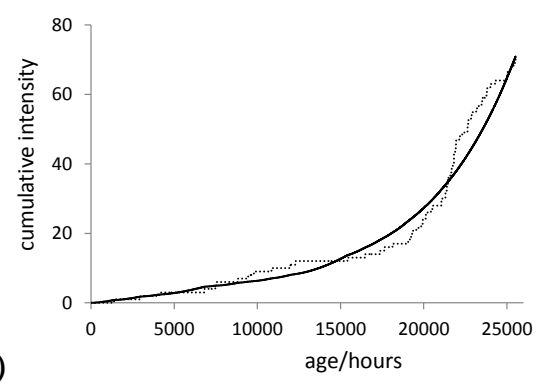

b)

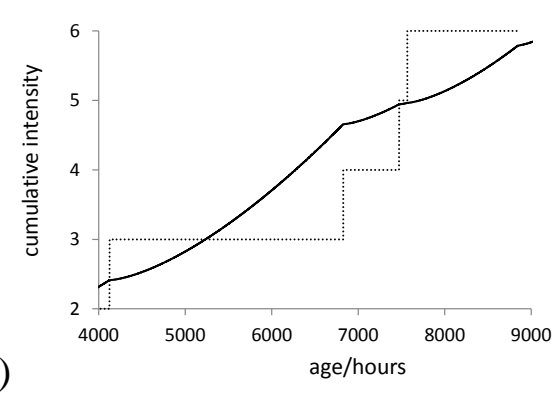

Figure 2. Halfbeak repair data: cumulative number of repairs (dotted line) and fitted (RP+NHPP) cumulative intensity function (solid line). a) all data; b) zoomed in.

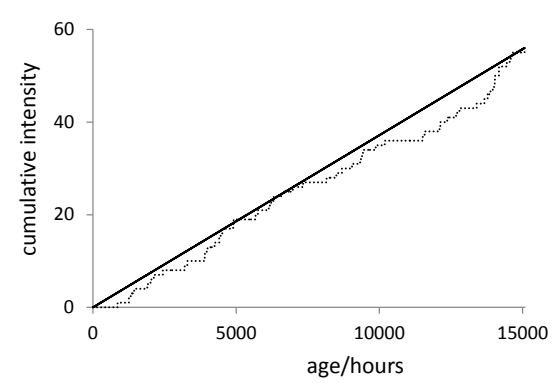

Figure 3. Grampus repair data: cumulative number of repairs (dotted line) and fitted (HPP) cumulative intensity function (solid line). 

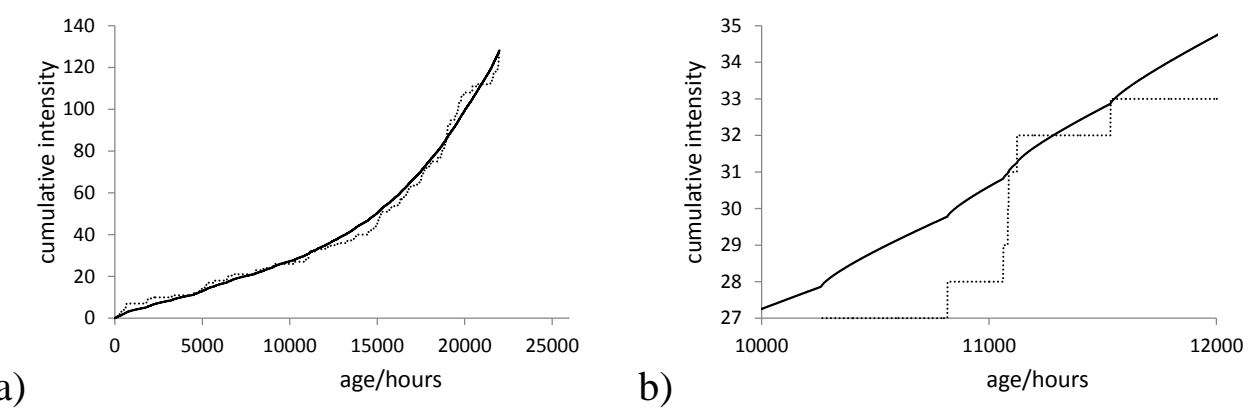

Figure 4. 180t dump-truck repair data: cumulative number of repairs (dotted line) and fitted (RP+NHPP) cumulative intensity function (solid line). a) all data; b) zoomed in.

The results for the 180t dump-truck are clear, like the Halfbeak. However, here the renewing part is DFR $\left(\hat{\beta}_{1}=0.69\right.$ ). This is apparent in Figure $4 \mathrm{~b}$, so that the system is at highest risk of failure immediately following a repair. The remainder of the system is also clearly ageing (Figure 4a).

The air-conditioner datasets we discuss collectively (Figure 5). On the whole these datasets are much smaller than the others, and therefore our conclusions should be more cautious. 7912 and 7914 show effects that are similar to the photocopier data: $\hat{\beta}_{1}$ very large for the minimum AIC model in each case, suggesting irregularity in the estimation (e.g. Cheng and Traylor, 1995). 7909 (an RP which is IFR, $\hat{\beta}_{1}=1.3$ ) is straightforward. The results for 7914 (the smallest dataset) for the models that are superpositions are suspect, and so in these circumstances we select the HPP (purely random repairs) as the best model.

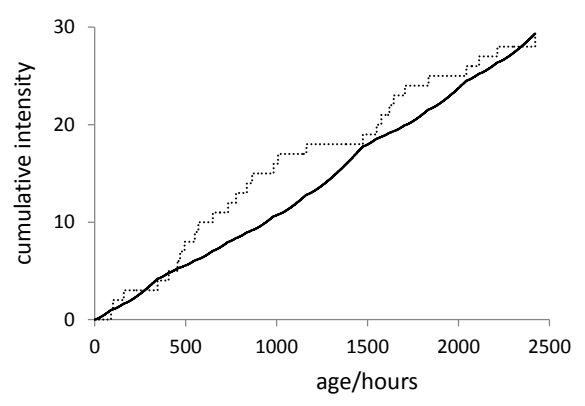

a) $7909, \mathrm{RP}$

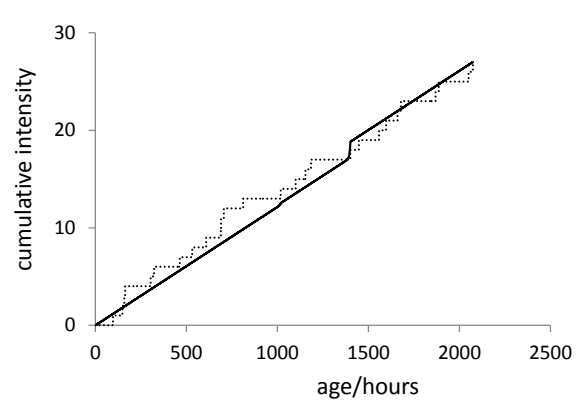

c) $7913, \mathrm{RP}+\mathrm{HPP}$

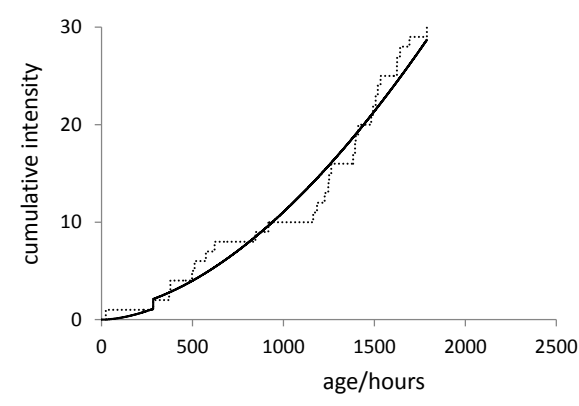

b) 7912, RP+NHPP

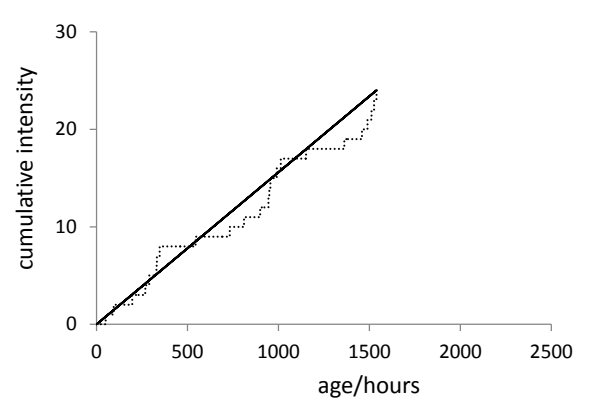

d) 7914, HPP

Figure 5. Air-conditioner repair data: cumulative number of repairs (dotted line) and fitted cumulative intensity function (solid line).

Throughout these analyses we use the mean time between repairs (MTBR) as a check on model fit, while noting that a fitted MTBR does not exist for a non-stationary model (NHPP or 
$\mathrm{RP}+\mathrm{NHPP})$. We can see that when estimation is irregular there is a tendency for the fitted MTBR to disagree with the observed MTBR.

Overall, we might conclude that 30 failures are is too few to fit models with more than two parameters. The RP+HPP has particular difficulties in this regard because it is stationary. Where the RP+NHPP fits well (Halfbeak and 180t dump-truck), the NHPP alone does not fit well. This supports the case for the usefulness of the models developed in this paper.

\section{Maintenance policy}

As we have so far not distinguished, both in the repair process modelling and the data fitting, preventive repairs from corrective repairs, we frame a maintenance policy in two ways: i) using a marginal-cost approach, and ii) as an age-based replacement policy (Barlow and Hunter, 1960).

\subsection{Marginal cost policy}

We consider a maintenance policy first in the context of the stationary model (4). Let us suppose that $h_{\mathrm{C}}($.$) is IFR. Then it is natural to subject the VS to age-based repair: that is, to preventively$ repair the VS (and hence the VC) when the time since last repair reaches some control limit, $T$, say. We call this the $T$-policy. Then we will notionally regard the efficacy of age-based repair as the repair effect. We use the notation $Z_{T}$ for the time between repairs under the $T$-policy, and note that $Z_{T}$ does not depend on the time of the last repair (because the VS is an RP). $X$ is the lifetime of the VC (with hazard function $\left.h_{\mathrm{C}}(x)\right) . Y \sim E x(\lambda)$ is the lifetime of the VSS. Then

$$
Z_{T}=\min (T, X, Y) \text {. }
$$

Definitions. (i) The marginal cost-rate $Q_{T}$ of the T-policy is $c_{\mathrm{P}} \phi_{\mathrm{P}}(T)$ where $c_{\mathrm{P}}$ is the cost of an additional, preventive repair and $\phi_{\mathrm{P}}(T)$ is the rate of occurrence of preventive repairs (preventiverepair-rate) under the T-policy. (ii) The preventive-repair effect is $\delta=100 \times \phi_{\mathrm{P}}(T) / \phi$, where $\phi$ is the rate of occurrence of (all) repairs (repair-rate).

The justification of these is as follows. A repair is either preventive or not preventive. Let us for this explanation call the latter event a failure. Then the preventive-repair effect is the percentage of failures prevented by preventive repair. If $T$ is small, most repairs will be preventive and so $\phi_{\mathrm{P}}(T) / \phi$ will be close to 1 , and the preventive-repair effect will be nearly $100 \%$. To counterbalance this, the marginal cost of the small $T$-policy will be high.

Proposition 4. Under the T-policy, $(i) \phi_{\mathrm{P}}(T)=\operatorname{Pr}\{\min (X, Y)>T\} / E\{\min (T, X, Y)\}$ and (ii) $\phi=1 / E\{\min (T, X, Y)\}$.

Corollaries. $\delta=100 \times \operatorname{Pr}\{\min (X, Y)>T\}$ and $\delta$ decreases as Tincreases.

Proofs. The corollaries follow immediately from the definitions and Proposition 4. Proposition 4(i) is true because the $T$-policy defines a renewal policy with renewal at the time (since last renewal) of the preventive repair, $T$, or "failure" of the VC, $X$, or "failure" of the VSS, $Y$, whichever occurs soonest, that is, at $Z_{T}=\min (T, X, Y)$. Therefore, the expected cycle length is $E\left\{Z_{T}\right\}$ and the probability that a renewal cycle ends with preventive-repair is $\operatorname{Pr}\{\min (X, Y)>T\}$. Defining $N_{\mathrm{P}}(\tau ; T)$ as the number of preventive repairs in $[0, \tau]$ under the $T$-policy, it follows that

$$
\phi_{\mathrm{P}}(T)=\lim _{\tau \rightarrow \infty} \frac{N_{\mathrm{P}}(\tau ; T)}{\tau}=\frac{\operatorname{Pr}\{\min (X, Y)>T\}}{E\left\{Z_{T}\right\}},
$$


by the renewal-reward theorem. Proposition 4(ii) follows by a similar argument in respect the number of repairs in $[0, \tau]$ under the $T$-policy, $N(\tau ; T)$. End of Proof.

For calculation of the terms in (11), we note that

$$
\operatorname{Pr}\{\min (X, Y)>T\}=\operatorname{Pr}(X>T) \operatorname{Pr}(Y>T)=S_{X}(T) S_{Y}(T),
$$

and

$$
\begin{aligned}
E\left\{Z_{T}\right\}=E\{\min (T, X, Y)\} & =\int_{0}^{T}\left\{1-F_{\min (X, Y)}(u)\right\} \mathrm{d} u \\
& =\int_{0}^{T} \operatorname{Pr}\{\min (X, Y)>u\} \mathrm{d} u \\
& =\int_{0}^{T} S_{X}(u) S_{Y}(u) \mathrm{d} u .
\end{aligned}
$$

\subsection{Cost-rate policy}

An alternative to the marginal cost approach is a total-cost approach. This may be used if the cost of preventive-repair, $c_{\mathrm{P}}$, and the cost of corrective repair, $c_{\mathrm{F}}$, are specified. Then, because the VS is an RP, the optimum $T$-policy is that which minimizes the long-run cost per unit time (cost-rate)

$$
Q_{\mathrm{ABR}}(T)=\frac{c_{\mathrm{P}} \operatorname{Pr}\{\min (X, Y)>T\}+c_{\mathrm{F}} \operatorname{Pr}\{\min (X, Y) \leq T\}}{E\left\{Z_{T}\right\}},
$$

and there exists an optimum policy, the $T^{*}$-policy, provided $h_{\mathrm{C}}($.$) is IFR and c_{\mathrm{P}}<c_{\mathrm{F}}$. This is just the age-based replacement policy when the random variable that describes the time to failure is $\min (X, Y)$.

Next we comment on the case when the VC is RP+NHPP (non-stationary). Our suggestion is to suppose that at some time $t^{\prime}$, at which the maintenance policy is being planned, $\lambda_{\mathrm{R}}(t)$ is fixed at least as far as until the next preventive-repair (quasi-stationary), and then to use either of the approaches above, planning preventive repairs one step at a time. In this way, effectively, a varying $\lambda_{\mathrm{R}}(t)$ is approximated by a step function (but is not fitted to data as such).

\subsection{Results}

We now implement this idea for the Halfbeak because RP+NHPP is the best model for this system and in this model $\beta_{1}>1$, so that preventive repair has a positive benefit (the system is better-thanold after repair). Note, when $\beta_{1}<1$, preventive repair has a negative benefit (the system is worsethan-old after repair).

For the Halfbeak, $X \sim \mathrm{We}\left(\alpha_{1}, \beta_{1}\right)$ so that $S_{X}(x)=\exp \left(-\left(x / \alpha_{1}\right)^{\beta_{1}}\right)$, and $Y \sim \operatorname{Ex}\left(\lambda_{\mathrm{R}}\right)$ so that $S_{Y}(y)=\exp \left(-\lambda_{\mathrm{R}} y\right)$. Under the quasi-stationary idea, we set $\lambda_{\mathrm{R}}=\left(\beta_{2} / \alpha_{2}\right)\left(t^{\prime} / \alpha_{2}\right)^{\beta_{2}-1}$. From (12) and (13) we then obtain that at time $t^{\prime}$

$$
\operatorname{Pr}\{\min (X, Y)>T\}=\exp \left\{-\left(T / \alpha_{1}\right)^{\beta_{1}}-\lambda T\right\}=\exp \left\{-\left(T / \alpha_{1}\right)^{\beta_{1}}-T\left(\beta_{2} / \alpha_{2}\right)\left(t^{\prime} / \alpha_{2}\right)^{\beta_{2}-1}\right\}
$$

and

$$
E\left\{Z_{T}\right\}=\int_{0}^{T} \exp \left\{-\left(u / \alpha_{1}\right)^{\beta_{1}}-u\left(\beta_{2} / \alpha_{2}\right)\left(t^{\prime} / \alpha_{2}\right)^{\beta_{2}-1}\right\} \mathrm{d} u \text {. }
$$

The parameters $\alpha_{1}, \alpha_{2}, \beta_{1}, \beta_{2}$ are set at their maximum likelihood estimates. We suppose that $c_{\mathrm{P}}=1$ and $c_{\mathrm{F}}=5$. 
In Figure 6a we present the preventive repair effect $\delta$, as we define it (Definition (ii) above), versus $T$ under the notion of quasi-stationarity. Thus we consider, hypothetically, what would be appropriate preventive-repair intervals when the system is aged $t^{\prime}$ hours, that is, at the time at which the system has been operating for $t^{\prime}$ hours, for $t^{\prime}=10000, t^{\prime}=20000$, and $t^{\prime}=30000$. These "ages" correspond roughly to early life, mature life, and beyond-useful operating life. The corresponding marginal cost-rates (assuming a unit cost for a preventive repair) are shown in Figure 6b. Figure 7 presents the total cost-rate, equation (14). Separate graphs are required here because the total cost-rates for the various $t^{\prime}$ are on very different scales. The total cost-rate at the optimum policy increases 5-fold at each age-step.

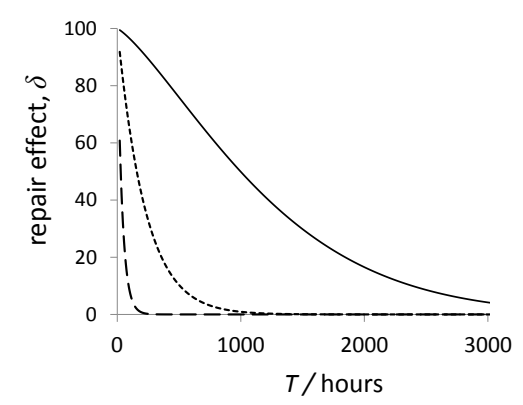

a)

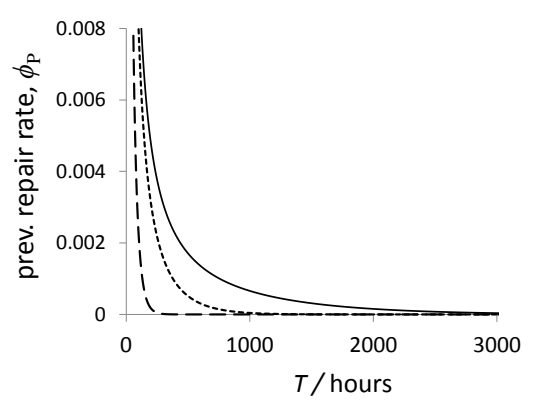

b)

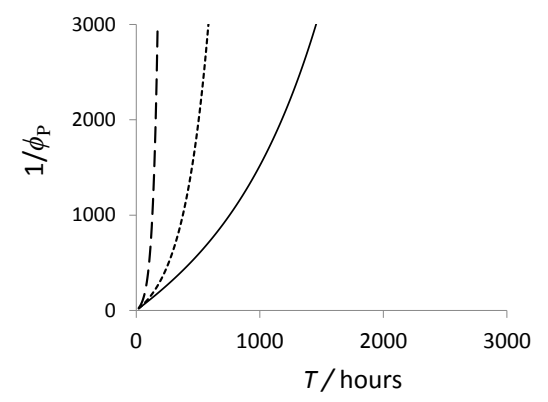

c)

Figure 6. Halfbeak: a) repair effect $\delta$ versus $T$, b) preventive-repair rate $\phi_{\mathrm{P}}(T)$ versus $T$, c) mean time between preventive repairs $1 / \phi_{\mathrm{P}}(T)$ versus $T$, under quasi-stationarity for $t^{\prime}=10000$ (solid line), $t^{\prime}=20000$ (dotted line), $t^{\prime}=30000$ (dashed line).

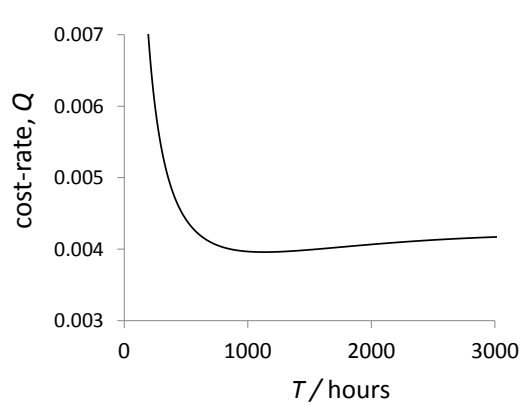

a) $t^{\prime}=10000$

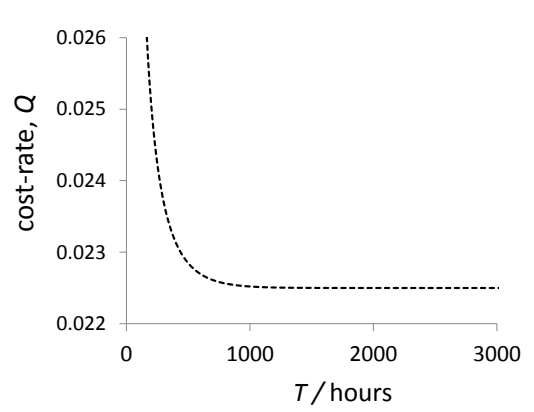

b) $t^{\prime}=20000$

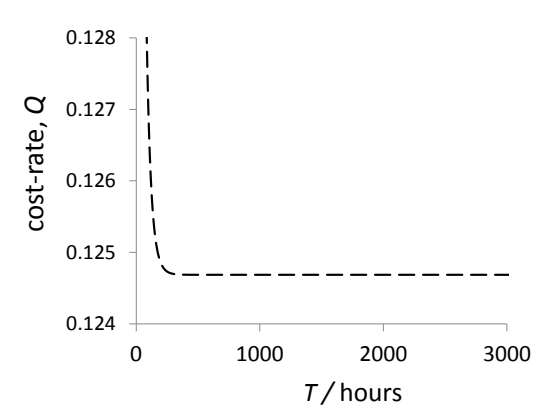

c) $t^{\prime}=30000$

Figure 7. Halfbeak: cost-rate $Q_{\mathrm{ABR}}(T)$ versus $T$ under a quasi-stationarity for a) $t^{\prime}=10000$ (solid line), b) $t^{\prime}=20000$ (dotted line); c) $t^{\prime}=30000$ (dashed line).

In Figure 6a) we can observe that, in the early life of the system, preventive-repair (in which the part that corresponds to the VC is renewed) at a 1000 operating-hours interval is approximately $50 \%$ effective. When the ratio of the cost-of-failure to cost of preventive-repair is 5 , the cost-rate is minimized at this interval (Figure 7a). In later life, because the part of the system that is not affected by preventive-repair is failing more frequently, preventive repair is now less effective $(<20 \%$ at $T=500$ hours) and preventive-repair is cost-inefficient (Figure $7 \mathrm{~b}$ ). Projecting beyond the actual lifetime of the system, we can see (Figure 6a and Figure 7c) that preventive maintenance is now hopeless in preventing failure. Figure 6c) confirms this analysis. When $t^{\prime}=10000$, for small $T$ 
( $<1000$ hours) nearly all repairs are preventive repair (noting that if $1 / \phi_{\mathrm{P}}(T)=T$ then every repair is a preventive repair). For $t^{\prime}=30000$, a $T$-policy even for a very small $T$ - which would have a very large cost —is almost completely ineffective.

\subsection{Implications for practice}

It is understood that in reality repair cannot make a system like-new, and industry has sought to quantify the effect of repair on a system. The models studied in this paper demonstrate partialrenewal, how the repair-effect can be quantified, and that repairs become less effective as a system ages, to a point where scrappage or complete overhaul is the only viable option. The models also quantify these notions, albeit once the system has been under observation for an extended period of operation. One might argue then this methodology is only useful for obsolete systems, and therefore never useful. We counter argue that the models will provide an environment (the mathematical world) in which to explore the behavior of new systems, for which data are not yet available, that are similar to those studied here. This paper then quantifies not only age-related partial-renewal but also shows - by considering a number of different systems - that systems differ in their ageing rates and in their balance of preventable to unpreventable failures and that maintenance needs to be adapted accordingly. The paper also indicates the typical quantity of objective data that is required for useful conclusions about system behavior to be drawn.

\section{Conclusions}

This paper describes some models of imperfect repair that are alternatives to age-reduction, hazardreduction, and geometric-type processes. The models use the concept of a virtual series system in which a virtual component is renewed whenever the system is repaired. Repairs may occur preventively or on failure of the virtual component or on failure of the remainder of the system. The system failure process is the superposition of the failure process of the virtual component and the failure process of the remainder. When the remainder fails it is minimally repaired (and the virtual component is replaced). This then models the notion that routine maintenance is carried out at every repair and that this routine maintenance is homogeneous and only marginally effective. The models developed can quantify the extent to which maintenance is effective and therefore be used in inform decision making about how often it should be scheduled.

The failure processes of the virtual component and the remainder of the system are modelled with counting processes, a renewal process for the former and a nonhomogeneous Poisson process for the latter. Two parameters is the minimum specification for each of these, making four in total for the full model. This is considerably less than would be required for a multi-component model. This (parsimonious parameterization) is the nature of the class of models that includes these virtual system models. It lends them to fitting to failure data, and we demonstrate this for a range of classic failure datasets. Therein, we find that reduced models are often best-fitting. Reduced models include the superposition of a renewal process and a homogeneous Poisson process corresponding to a renewing part and a non-ageing part (three parameters), a nonhomogeneous Poisson process corresponding to a system with no renewing part (two parameters), a renewal process corresponding to a renewing system (two parameters), and a homogeneous Poisson process corresponding to a non-ageing system with no renewing part (one parameter). The best-fitting model then indicates which particular maintenance policy is appropriate. 
A model with few parameters is important because failures are rare. Indeed extant systems for which the maintenance requirements must be specified may not have failed ever. Nonetheless, engineers can make judgements about whether extant systems are similar in their failure behaviour to legacy systems and use the modelling of legacy systems to inform decisions. Where some failures have been observed this suggests that estimation of the models described in this paper might proceed through a Bayesian approach, whereby fitted models for legacy systems are used to encode prior information about parameters. We will consider the efficacy of such an approach in further development of this work. Another development that can be explored in future is fitting the models to data with right censoring (survivals). The log-likelihood (7) lends itself directly to this. Analysis of model for software reliability would also be interesting.

The implications for practice are as follows. Firstly, these models can be used to estimate repair-effects indirectly and objectively. Secondly, if repair is such that its effect is to renew one ageing part of a system, then more spending on repairs is beneficial, and if a particular benefit (preventive-repair-effect) is desired then its cost may be determined. Alternatively, a costminimizing repair policy may be determined provided the cost of preventive-repair is less than the cost of corrective repair and the repairable part is ageing. If the remainder is ageing, then the maintenance policy needs to be adapted as it ages.

\section{References}

Akaike H (2011) Akaike's Information Criterion. In International Encyclopaedia of Statistical Science. Springer, Berlin.

Akbarov A, Christer AH, Wang W (2008) Problem identification in maintenance modelling: a case study. International Journal of Production Research 46, 1031-1046.

Akman VE, Raftery AE (1986) Bayes Factors for Non-Homogeneous Poisson Processes with

Vague Prior Information. Journal of the Royal Statistical Society, Series B 48, 322-329.

Andersen P, Borgan O, Gill R, Keiding N (1993) Statistical Models Based on Counting Processes. Springer, New York.

Ascher H, Feingold (1984) Repairable Systems Reliability. Marcel Dekker, New York.

Baker RD (1991) Testing the efficacy of preventive maintenance. Journal of the Operational Research Society 42, 493-503.

Baker RD (1996) Some New Tests of the Power Law Process. Technometrics 38, 256-265.

Baker RD, Scarf PA (1995) Can models fitted to small data samples lead to maintenance policies with near-optimum cost?, IMA Journal of Mathematics Applied in Business \& Industry 6, 3-12.

Barlow RE, Hunter LC (1960) Optimum preventive replacement policies. Operations Research 8, 90-100.

Barlow RE, Proschan F, Hunter LC (1996) Mathematical Theory of Reliability. SIAM, Philadelphia.

Blischke WR, Murthy DNP (2000) Reliability: Modeling, Prediction and Optimization. Wiley, New York.

Brown M, Proschan F (1983) Imperfect repair. Journal of Applied Probability 20, 851-859.

Caroni C (2010) "Failure limited" data and TTT-based trend tests in multiple repairable systems. Reliability Engineering \& System Safety 95, 704-706.

Cheng R, Traylor L (1995) Non-regular maximum likelihood problems. Journal of the Royal Statistical Society. Series B 57, 3-44. 
Coetzee JL (1996) Reliability degradation and the equipment replacement problem. In: Proc. Int. Conf. of Maintenance Societies (ICOMS-96). Melbourne, Paper 21.

Cox DR, Lewis PAW (1966) Statistical Analysis of a Series of Events. Methuen, London.

Crowder, M. (2012). Multivariate Survival Analysis and Competing Risks. Chapman and Hall, New York.

Crowder M, Kimber AC, Smith RL, Sweeting TJ (1991) Statistical Analysis of Reliability Data. Chapman and Hall, London

Desa MI, Christer AH (2001) Modelling in the absence of data: a case study of fleet maintenance in a developing country. Journal of the Operational Research Society 52, 247-260.

Do P, Assaf R, Scarf PA, Iung B (2018) Condition based maintenance for a multi-component system with stochastic and economic dependencies. Reliability Engineering \& System Safety (in press).

Doyen L (2014) Semi-parametric estimation of Brown-Proschan preventive maintenance effects and intrinsic wear-out. Computational Statistics \& Data Analysis 77, 206-222.

Doyen L, Gaudoin O (2004) Classes of imperfect repair models based on reduction of failure intensity or virtual age. Reliability Engineering \& System Safety 84, 45-56.

Doyen L, Gaudoin O, Syamsundar A (2017) On geometric reduction of age or intensity models for imperfect maintenance. Reliability Engineering \& System Safety 168, 40-52.

Fuqing Y, Kumar U (2012) A general imperfect repair model considering time-dependent repair effectiveness. IEEE Transactions on Reliability 61, 95-100.

Gámiz ML, Lindqvist BH(2016) Nonparametric estimation in trend-renewal processes. Reliability Engineering \& System Safety 145,38-46.

Hong W-C, Pai P-F (2006) Predicting engine reliability by support vector machines. The International Journal of Advanced Manufacturing Technology 28, 154-161.

Jack N (1998) Age-reduction models for imperfect maintenance. IMA Journal of Management Mathematics 9, 347-354.

Kallen MJ (2011) Modelling imperfect maintenance and the reliability of complex systems using superposed renewal processes. Reliability Engineering \& System Safety 96, 636-641.

Kijima M, Sumita U (1986) A useful generalization of renewal theory: Counting processes governed by non-negative Markovian increments. Journal of Applied Probability 23, 71-88.

Kijima M (1989) Some results for repairable systems with general repair. Journal of Applied Probability 26, 89-102.

Lam Y (1988) Geometric processes and replacement problem. Acta Mathematicae Applicatae Sinica 4, 366-377.

Lawless JF (1983) Statistical methods in reliability. Technometrics 25, 305-355.

Lee L (1980) Testing adequacy of the Weibull and log-linear rate models for a Poisson process. Technometrics 22, 195-200.

Meeker WQ, Escobar LA (1998) Statistical Methods for Reliability Data. Wiley, New York.

Percy DF, Alkali BM (2006) Generalized proportional intensities models for repairable systems. IMA Journal of Management Mathematics 17, 171-185.

Percy DF, Kearney JR, Kobbacy KAH (2010) Hybrid intensity models for repairable systems. IMA Journal of Management Mathematics 21, 395-406.

Phillips MJ (2000) Bootstrap confidence regions for the expected ROCOF of a repairable system. IEEE Transactions on Reliability 49, 204-208. 
Phillips M J (2001) Estimation of the expected ROCOF of a repairable system with bootstrap confidence region. Quality Reliability Engineering International 17, 159-162.

Proschan F (1963) Theoretical explanation of observed decreasing failure rate. Technometrics 5, 375-383.

Pulcini G (2001) Modeling the failure data of a repairable equipment with bathtub type failure intensity. Reliability Engineering \& System Safety 71, 209-218.

Rigdon SE, Asit P (1989) The Power Law Process: A Model for the Reliability of Repairable Systems. Journal of Quality Technology 21, 251-260.

Ross S (1996) Stochastic Processes. Wiley, New York.

Scarf PA (1997) On the application of mathematical models in maintenance, European Journal of Operational Research 99, 493-506.

Scarf PA, Cavalcante CAV (2012) Modelling quality in replacement and inspection maintenance. International Journal of Production Economics 135, 372-381.

Smith MAJ, Dekker R (1997) Preventive maintenance in a 1 out of n system: The uptime, downtime and costs. European Journal of Operational Research 99, 565-583.

Syamsundar A, Kumar DEV (2017) Geometric failure rate reduction model for the analysis of repairable systems. In Annual Reliability and Maintainability Symposium 2017, Orlando, pp.14.

Tanwar M, Rai RN, Bolia N (2014) Imperfect repair modeling using Kijima type generalized renewal process. Reliability Engineering \& System Safety 124, 24-31.

Vaurio JK (1999) Identification of process and distribution characteristics by testing monotonic and non-monotonic trends in failure intensities and hazard rates. Reliability Engineering \& System Safety 64, 345-357.

Wang H, Pham H (1996) A quasi renewal process and its applications in imperfect maintenance. International Journal of Systems Science 27, 1055-1062.

Wu S, Clements-Croome D (2006) A novel repair model for imperfect maintenance. IMA Journal of Management Mathematics 17, 235-243.

Wu S, Scarf PA (2015) Decline and repair, and covariate effects. European Journal of Operational Research 244, 219-226.

Wu S, Scarf PA (2017) Two new stochastic models of the failure process of a series system. European Journal of Operational Research 257, 763-772.

Wu S, Wang G (2018) The semi-geometric process and some properties. IMA Journal of Management Mathematics 29, 229-245.

Zhang M, Gaudoin O, Xie M (2015) Degradation-based maintenance decision using stochastic filtering for systems under imperfect maintenance. European Journal of Operational Research 245, 531-541.

Zhang W, Wang W (2014) Cost modelling in maintenance strategy optimisation for infrastructure assets with limited data. Reliability Engineering \& System Safety 130, 33-41. 